\title{
Evaluation of article structure components in English-Kwanyama Dictionary (EKD) with reference to the target users
}

\author{
Edward Shikesho ${ }^{1}$ \\ Ministry of Education, Arts and Culture
}

\begin{abstract}
This paper evaluates the article structure components in English-Kwanyama Dictionary (EKD) with reference to the target users. For any bilingual dictionary to satisfy the needs of the target users, it should be user-friendly. Important aspects that have to be considered when one compiles a bilingual dictionary are, among others, orthography, pronunciation, inflections, parts of speech, and translation equivalents. All these aspects are all parts of the microstructure (Gouws, 2002; Gouws \& Prinsloo, 2005; Svensén, 2009). A target user should also be defined in any bilingual dictionary, and should be indicated in the dictionary's front matter texts (Gouws \& Prinsloo, 2005). EKD was compiled in 1954, about four and half decades ago, and there is no comprehensive research done to analyse its quality. The study's theoretical frameworks are the Function Theory and the Text theory. Sven Tarp and Henning Bergenholtz are the leading proponents of the Function theory which was established in the late 1980s, and deals with dictionary functions and the user needs. The Text theory, proposed by Herbert Ernst Wiegand in the 1990s, deals with dictionaries' textual structures. It is a qualitative study in a form of text analysis. Systematic dictionary research, which consists of two types, functional text segmentation as well as philological methods, is adopted. The findings reveal that EKD has become old and much needs to be improved in terms of aspects such as orthography. EKD's target users are not defined in the dictionary's front matter text, therefore, this paper recommends that lexicographers should define their target users in their future dictionaries' front matter texts. They should also take into consideration the correct presentation of article structure aspects, based on the comment on form and the comment on semantics, such as spelling, parts of speech indication, lemma inflection, morphology and translation equivalents.
\end{abstract}

\section{Introduction}

This study is based in the field of theoretical lexical lexicography that deals with research on dictionaries. The purpose is to evaluate the types of microstructure in Engish-Kwanyama Dictionary $(E K D)$ with reference to the target users. EKD was compiled by two missionaries who settled at Oukwanyama between 1924 and 1947. G.W.R. Tobias founded St. Mary's Mission at Odibo. B.H.C. Turvey worked at Holy Cross Mission District at Onamunhama, as a Reverend. The dictionary was later published in 1954 by the Witwatersrand University, South Africa.

Dictionary history, dictionary criticism, dictionary typology, dictionary structure, dictionary use and the dictionary IT are "perspectives" identified by Hartmann (2013) in metalexicographic research. While Hartmann (2013) calls them perspectives, Wiegand (1984) names them components, as

\footnotetext{
${ }^{1}$ Edward Shikesho is a high school teacher in the Ministry of Education, Arts and Culture. He teaches English as a Second Language and Oshikwanyama as a First Language (Grade 8-12) at Nuuyoma Secondary School in Omusati Region, Namibia. He holds a Bachelor of Education (Honours) degree and a Master of Arts in African Languages (Oshiwambo Studies) from the University of Namibia (UNAM) in 2015 and 2019, respectively. While he was an undergraduate student, he served as a writing tutor in the Writing Excellence Unit, at the UNAM Language Centre. His research interests include Oshikwanyama lexicography, linguistics, and Oshiwambo cultural practices. Email: eshikesho@yahoo.com
} 
they comprise dictionary research. These components, according to Wiegand (1984) are user research, dictionary criticism, and history of lexicography and the general theory of lexicography. This study falls under "dictionary criticism", because it aims to evaluate the types of microstructure in EKD with reference to the target users.

Gouws and Prinsloo (2005) assert that a dictionary's article structure is made up of two prime types, the comment on form and the comment on semantics. The comment on form is the search field in which all the types of data that have to do with the form of the lemma sign are accommodated. These data types include, among others, phonetics, orthographic forms, pronunciation, morphological data, and parts of speech. The lemma sign is also regarded as part of the comment on form because of the information on the spelling of the relevant lexical item that it provides.

The comment on semantics is the search field in which all the data types that concern and reflect semantic as well as pragmatic features of the lexical item which is presented by the lemma are accommodated. These data types are definitions or translation equivalents, etymological data, lexicographical labels, co-text entries and context and other data such as inserted inner texts (Gouws \& Prinsloo, 2005).

This study only focuses on pronunciation, part of speech, lemma inflection as parts of the comment on form, and translation equivalents, as parts of the comment on semantics.

This study is important because, through it, future scholars and researchers in the field of theoretical lexicography will be assisted in compiling and establishing higher quality bilingual dictionaries that will assist in developing the Oshikwanyama lexicography, which seems to be in its infancy stage.

\section{Literature Review Evaluation of dictionaries}

Dictionary evaluation is a significant element in theoretical lexicography. Every lexicographical work has its unique characteristics and structures. Therefore, it is important that it is evaluated. This section focuses on criteria for evaluating a dictionary as proposed by different scholars in the field of theoretical lexicography.

Due to the fact that different types of dictionaries are consulted by different users, it is also important for them to be evaluated to assess their appropriateness. Jackson (2002) clarifies that many dictionaries are published for commercial purposes. It is believed that various publishers do invest a reasonable amount of money in their development, and they are tailored to perceived market needs. Therefore, there is a need to select the criteria for evaluating a certain lexicographical work.

The main communicative objective of dictionary evaluation, according to Swanepoel (2013), is to give lexicographers guidelines on how to deal with the improvement of their dictionaries' functional quality. Swanepoel (2013) defines dictionary evaluation as follows: "Dictionary evaluation as praxis, involves the analysis, description and evaluation of the design features of a dictionary on the basis of one or more lexicographically relevant evaluation criteria" (p. 587). In expansion of the preceding definition by Swanepoel (2013), dictionary evaluation is viewed as a practical activity that has to do with analysis, descriptions and evaluation of various features of dictionaries based on different criteria. 
Dictionaries are generally used for information such as translation equivalents, spellings of words, pronunciation, etymology and usage of words, regional usage of words, synonyms, and antonyms, just to mention but a few; therefore, these aspects need to be taken into consideration when one evaluates a dictionary (Klapicová, 2005).

Svensén (2009) agrees with Klapicová (2005), and explains that a dictionary should be evaluated on the basis of the qualities that it is asserted by the publisher to possess. It is therefore a relevant and crucial procedure to first start by mentioning the target user groups and functions the dictionary is produced for, the type of information it contains and the properties the dictionary is believed to have. However, Svensén (2009) further establishes the idea that a large number of dictionaries fail to provide this information, and in cases such as this, the evaluation will only depend on general criteria that are in fact adopted, that are independent of the dictionary which is under review.

Svensén (2009, p. 483) further suggests a list of 32 aspects that have to be taken into consideration when one evaluates a dictionary. These aspects are "dictionary functions, dictionary users, advice given to the users, price, layout / web design, the compilers, comparison with other dictionaries, prehistory of the dictionary, reference to other reviewers, the reviewer, dictionary basis, outside matters, lemma selection, establishment of lemma, search and access options, entry structure, the normative/ descriptive dimension, equivalents, grammar, orthography, pronunciation, semantic and encyclopaedic information, diasystematic information, etymology, examples, collocations, idioms, illustrations, synonymy/ antonym, cross-reference, entertainment value, and a unified concluding evaluation".

Jackson (1996), also proposes criteria for evaluation of dictionaries, and according to him, the main criteria are vocabulary, word formation, homographs, definitions, lexical relations, pronunciation, grammar, usage, examples, etymology, special features and criticism. Bergenholtz and Gouws (2016) advise reviewers that anyone reviewing a dictionary should be fair. Although it is believed that publishing houses expect positive comments from reviewers, negative comments can also assist compilers to improve future dictionaries. Furthermore, any type of dictionary, whether it is good or bad, is a tool that a user uses in a real situation; therefore, it should be evaluated.

Among the above criteria as discussed by various scholars, this study only focuses on target users, pronunciation, spelling, morphology and inflection, and translation equivalents. It is therefore worth emphasising that dictionary evaluation as a practical activity is regarded as very important in a sense that it does not only provide informed reviews to potential users about dictionaries, but it also leads to further development in the field of theoretical lexicography and to improvements in future dictionaries.

\section{Theoretical Framework \\ The Function Theory}

The Function Theory, also known as The Modern Theory of Lexicographic Functions, was developed by scholars at the Centre for Lexicography at the Aarhus School of Business in Denmark since the late 1980s (Tarp, 2008). Two scholars, Sven Tarp and Henning Bergenholtz, are the leading proponents of the Function Theory. According to Tarp (2008) and Tarp (2013), four main components of the Function Theory namely, user, user needs, user situation and the assistance that dictionaries give to satisfy the user needs are identified. It is further added that these four components are interconnected and therefore, need to be analysed in a separate way and then they need to be compared with other components to make it possible for synthesising the elements that are identified by analysis (Tarp, 2008). 
According to Tarp (2008), the first three of the four elements, user, user situations and user needs, are regarded as the starting point for both theoretical and practical lexicography.

\author{
The user \\ According to Tarp (2013), the potential dictionary user is the focus of the Function theory, even \\ though it also does not ignore or reject the actual user. Lexicographers need to determine the \\ type of intended user group as well as a type of the user situations to which needs or problems \\ may be identified that can easily be covered when lexicographical data is provided, so that the \\ functions as well as the dictionary's genuine purpose can easily be established (Bergenholtz \& \\ Tarp, 2003). \\ In order to have a good understanding of the potential dictionary user, the Function theory, \\ according to Tarp (2013), establishes a typology of relevant user characteristics. The following is \\ a list of lexicographically relevant criteria in terms of user characteristics as provided by Tarp \\ (2008, p. 55) and Tarp (2013, p. 463): \\ - What is the mother tongue of the users? \\ - To what extent do they master their mother tongue? \\ - To what extent do they master a specific foreign language? \\ - To what extent do they master a specific specialised language in their mother tongue? \\ - To what extent do they master a specific specialised language in a foreign language? \\ - How much experience of translation do they have? \\ - How great is their general cultural knowledge? \\ - How great is their knowledge of culture in specific foreign language area? \\ - How much do they know about a specific subject or science?
}

Apart from the nine characteristics given above, there are also some relevant characteristics, for instance, lexicographical qualifications, which are activated when users change from being potential dictionary users to being actual dictionary users when consulting a dictionary, thereby generating a new kind of need aimed at finding and interpreting the lexicographical data that has been gathered and structured in the dictionary. Therefore, Tarp (2013, p. 464) has summarised the criteria for defining these qualifications into three questions as follows:

- How much do users know about lexicography?

- What general experience of dictionary usage do they have?

- What specific experience do they have of specific dictionary?

Based on the nine questions plus the other three provided above, Tarp (2013 p. 464) has established the fact that answers to them "make it possible to define the most important characteristics of potential users, and thereby to classify the various types of user."

\title{
The user situation
}

The Function Theory also focuses on the user situation, where lexicographically relevant needs may arise that may lead to an actual dictionary-usage situation (Tarp, 2013). In this regard, a distinction is made between two different types of user situation, namely, communicative situation and cognitive situation. The cognitive situations are related to an area of knowledge which is fixed and which is not so complicated to study, while the communicative approach is a little bit more complex. There are many changing factors to take into consideration when any communicative situation prevails, for example, the sender-receiver model (Wang, 2012). As far as communicative situation is concerned, potential users may run into problems that have to be solved in order to 
guarantee a successful communication process, i.e. text reception, text production or translation. In a cognitive situation, potential users, for one reason or the other, may wish or need to increase their knowledge about some subject or topic (Tarp, 2013).

The user needs

User needs, according to Tarp (2013), are sub-divided into two main groups. The first group is primary user needs, which are referred to as needs that lead to the usage situation of a dictionary, and the second group is the secondary user needs, which are referred to as needs that develop when users search for help in a dictionary. Tarp (2013, p. 465) explains that "primary user needs are always needs for information, which can be used to solve problems or gain knowledge. Secondary needs are needs for particular information, needs for instruction as well as education in dictionary usage."

Lexicographical assistance

The assistance from lexicographical works is what assists in the fulfilment of the needs mentioned in the previous sub-section. Tarp (2013) explains that the assistance that dictionaries can give for users is made up of lexicographical data that the users can obtain information from, to cover and solve their problems in specific situations. The satisfaction of user needs in such a way is referred to as the dictionary's function(s).

The Function Theory is a significant theory in theoretical lexicography as it gives lexicographers ideas, functions and purposes of dictionaries. The Function Theory is applied in this study in order to investigate its principles, its elements and its functions, that will be applied in evaluating EKD.

\section{The Text Theory}

The Text Theory is also important to this study because it is used in studying and evaluating EKD's textual structures. According to Tarp (2008), one of the most influential and active researchers in this field was Herbert Ernst Wiegand. Wiegand established the Text Theory or The Theory of Lexicographical Texts at the University of Heidelberg, Germany in the 1990s on the basic principle that dictionary articles are texts (Müller-Spitzer, 2013; Beyer \& Augart, 2017). Wiegand developed this theory based on his belief that dictionaries should be useful. His investigations into dictionaries as well as dictionary articles have helped lexicographers to understand the different textual structures in dictionaries and dictionary articles.

The components of dictionary article structure

Based on the Text Theory, Gouws and Prinsloo (2005) assert that a dictionary's aerticle structure consists of two major article components. These components are the comment on form and the comment on semantics. The former is the search field in which all the types of data that have to do with the form of the lemma sign are accommodated. These data types include, among others, phonetics, orthographic forms, pronunciation, morphological data, and parts of speech. It is also worth pointing out that the lemma sign is part of the comment on form in a sense that it gives information on the spelling of the relevant lexical item.

The comment on semantics is the search field in which all the data types that concern and reflect semantic as well as pragmatic features of the lexical item which is presented by the lemma are accommodated, such as definitions or translation equivalents, etymological data, lexicographical labels, co-text entries (words that precede or come after a given lexical item, that assist in the understanding of the meaning) and context entries (the information about the given lexical item) and other data such as inserted inner texts (Gouws \& Prinsloo, 2005).

This sub-section briefly presents elements that are part of the article structure types, namely, the comment on form and the comment on semantics, as discussed by several scholars: 
Spelling (Orthography)

Spelling is part of the comment on form based on the text theory, according to Gouws and Prinsloo (2005). The normal spelling is indicated by the lemma sign, but any variations will follow as variant of lemma signs. Gouws and Prinsloo (2005) further add that orthographical guidance is very salient because it helps users to find the lemma sign as well as to retrieve the necessary information on spelling of a certain lexical item. Extra spelling information or guidance is accommodated, especially when any given lexical item which is functioning as a lemma has spelling variants.

\section{Pronunciation}

Pronunciation is also part of the comment on form. This is usually within brackets [ ] or slashes // in conjunction with any variations. Gouws and Prinsloo (2005) write that various methods of presenting pronunciation in dictionaries prevail. These methods are different when it comes to the amount of pronunciation guidance a dictionary article offers. Some dictionaries can provide comprehensive phonetic transcription using the symbols from the International Phonetic Alphabet, while others indicate a partial transcription of the word or rather "only an orthographic transcription, trying to capture the word in the ordinary writing system" (Gouws \& Prinsloo, 2005, p. 119).

\section{Inflections}

Formation of words is the prime focus of this element. Existing words may change depending on their grammatical meaning, for example plurals, past tense, and so on Furthermore, some spelling adjustments here have to be indicated, such as doubling of consonants, and dropping of sounds, such as [e], or changing the glide [y] to [i]. Gouws and Prinsloo (2005) also assert that entries that indicate morphological data such as plurals as well as diminutives are also accommodated.

\section{Part of speech}

What plays a dominant role here when it comes to indication of this part of the microstructure is conventional abbreviations. The abbreviation such as adj. usually stands for 'adjective', $n$. stands for 'noun', and adv. stands for 'adverb'. Verbs are also marked v.t. if they are transitive and if they are intransitive they are marked as v.i.

\section{Translation equivalents}

Translation equivalents, according to Gouws and Prinsloo (2005), are part of the comment on semantics. Translation equivalents are the main foci of bilingual dictionaries. Modjadji (2012) clarifies that structural markers that are frequently used, as far as translation equivalents are concerned, are a comma (,) and a semi-colon (;). A comma separates synonyms and partial synonyms (lexical items that replace each other in all occurrences) and a semicolon separates polysemous senses, which are lexical items which cannot replace each other in any context. Every translation equivalent can stand on its own due to its different meaning. This study then adopts the numbering style of separating polysemous senses, for the user to simply get the information quickly and with ease.

\section{Evaluation}

\section{Target user profile}

The target user has not been defined in EKD's outer texts. Therefore, the target user profile is constructed based on answers to the questions of $\operatorname{Tarp}(2008,2013)$ that are relevant to EKD as provided below:

(a) What is the mother tongue of the target users? 
For $E K D$, two distinct potential target user groups can be distinguished, namely mother-tongue speakers of English and mother-tongue speakers of Oshikwanyama. Each group is given an index to facilitate future reference:

$E(U G)=$ Target user group consisting of mother-tongue speakers of English .

$\mathrm{O}(\mathrm{UG})=$ Target user group consisting of mother-tongue speakers of Oshikwanyama.

(b) To what extent do they master their mother-tongue?

Mother-tongue competence of $E(U G)$ is designated as $L 1_{E}$, and that of $O(U G)$ as $L 1_{O}$.

$\mathrm{L} 1_{\mathrm{E}}=$ Given that instruction in and through the medium of English is readily available internationally and in Namibia, members of $E(U G)$ are expected to have the following $L 1$ competencies:

$\mathrm{L} 1_{\mathrm{E}-1}=$ completely literate

$\mathrm{L} 1_{\mathrm{E}}-2=$ advanced competence in English grammar

$\mathrm{L} 1_{\mathrm{E}}-3=$ advanced competence in English lexicon

$\mathrm{L} 1_{\mathrm{E}}-4=$ advanced competence in English orthography

$\mathrm{L} 1 \mathrm{O}=$ Due to more limited instruction and availability of literature in their mother-tongue (medium of instruction up to Grade 3, after that Oshikwanyama is offered as optional language subject up to tertiary level), members of $\mathrm{O}(\mathrm{UG})$ are expected to have the following $\mathrm{L} 1$ competencies:

$\mathrm{L} 1 \mathrm{O}-1$ = literate to an advanced degree

L1o-2 = intermediate competence in Oshikwanyama grammar

L1o-3 = intermediate competence in Oshikwanyama lexicon

L1o-4 = intermediate to basic competence in Oshikwanyama orthography

(c) To what extent do they master the relevant foreign language?

For $E(U G)$, Oshikwanyama is regarded as the foreign language $(F L)$, and for $O(E G)$, English is regarded as the FL. FL competence of $E(U G)$ is designated as $F L_{E}$, and that of $O(U G)$ as $F L_{O}$. $F L_{E}=$ Given that no instruction in Oshikwanyama is generally available for $E(U G)$, members of $E(U G)$ are expected to have the following FL competencies in Oshikwanyama:

$\mathrm{FL}_{\mathrm{E}}-1$ = literate to the limited extent that texts can be read without comprehension and with partially correct pronunciation because the same alphabet is used in English

$F L_{E}-2$ = zero competence in Oshikwanyama grammar

$\mathrm{FL}_{\mathrm{E}}-3$ = zero competence in Oshikwanyama lexicon

$\mathrm{FL}_{\mathrm{E}}-4$ = zero competence in Oshikwanyama orthography

$F L_{0}=$ Given that instruction in English starts only at school and English is generally not spoken at home, members of $\mathrm{O}(\mathrm{UG})$ are expected to have the following FL competencies in English:

FLo-1 = intermediate literacy in English

FLo-2 = basic to intermediate competence in English grammar

$\mathrm{FL}_{\mathrm{O}}-3$ = basic to intermediate competence in English lexicon

FLo-4 = basic to intermediate competence in English orthography

(d) How great is their general cultural knowledge?

General cultural knowledge for $E(U G)$ is designated as $C_{E}$ and that of $O(U G)$ is designed as $\mathrm{CK}_{\mathrm{O}}$.

$\mathrm{CK}_{\mathrm{E}}-1=$ advanced to expert knowledge of interacting with one another 
$\mathrm{CK}_{\mathrm{E}}-2=$ advanced to expert knowledge of writing and reading texts in their mother tongue

$\mathrm{CK}_{\mathrm{O}-1}=$ advanced to expert knowledge of interacting with one another

$\mathrm{CK}_{\mathrm{O}-2}=$ intermediate knowledge of writing and reading texts in their mother tongue

(e) How great is their knowledge of the FL culture?

The knowledge of the FL culture for $E(U G)$ is designated as $F L C_{E}$ and that of $O(U G)$ is designated as $\mathrm{FLC}_{\mathrm{O}}$.

$\mathrm{FLC}_{\mathrm{E}}-1=$ zero knowledge of Oshikwanyama culture

$\mathrm{FLC}_{\mathrm{E}}-2=$ zero to basic knowledge of Oshikwanyama culture

FLCo-1 = basic to intermediate knowledge of (international) English/Western culture

FLCo-2 = basic to intermediate knowledge of Western culture

(f) What general experience of dictionary use do they have?

The general experience of dictionary use for $E(U G)$ is designated as $D U_{E}$ and that of $O(U G)$ is designated as DU。

$D U_{E}=$ Fair, because of a comparatively better reading culture and access to English dictionaries and their use

$\mathrm{DU}_{\mathrm{E}-1}=$ advanced experience of how to find a lemma they are looking for

$\mathrm{DU}_{\mathrm{E}}-2$ = advanced knowledge of how lemmata are arranged such as strict alphabetical vertical macrostructural ordering

$\mathrm{DU}_{\mathrm{E}}-3$ = advanced knowledge of finding translation equivalents

$\mathrm{DU}_{\mathrm{O}}=$ Very limited experience due to a low reading culture and almost no access to Oshikwanyama dictionaries or dictionary use experience

$\mathrm{DU}_{\mathrm{O}}-1$ = limited experience of how to find a lemma they are looking for

$\mathrm{DU}_{\mathrm{O}-2}=$ limited experience of how lemmata are arranged, for instance, strict alphabetical vertical macrostructural ordering

$\mathrm{DU}_{\mathrm{O}}-3$ = limited knowledge of finding translation equivalents

(g) What specific experience do they have of using EKD?

The specific experience of using EKD for $E(U G)$ is designated as $S E_{E}$ and that of $O(U G)$ is designated as SEO.

$\mathrm{SE}_{\mathrm{E}}-1=$ zero experience in using $E K D$

$\mathrm{SE}_{\mathrm{O}-1}=$ zero to limited experience in using $E K D$

Among the nine target user questions that Tarp $(2008,2013)$ provides, the above seven have been selected for EKD and are considered relevant.

\section{Article structure components}

The following microstructural elements are evaluated based on the comment on form as well as the comment on semantics as two components of the article structure of a dictionary as based on the Text theory.

\section{The comment on form}

The comment on form consists of four elements. The lemma sign, data on part of speech, data on inflection and data on pronunciation.

\section{The lemma sign}


The evaluation is done on the two types of lemmata: main lemmata and sub-lemmata. The first analysis is done on the main lemmata.

Main lemmata

\author{
Description \\ Lemma signs throughout the dictionary are spelt correctly. That means the target users, \\ particularly the mother-tongues speakers of Oshikwanyama will not be misguided or learn \\ incorrect spelling in the source language. Apart from spelling, it can also be indicated that all the \\ lemma signs are bolded (typographical structural marker). This is done to assist the target users \\ to identify them easily when they are looking for them in the dictionary. Compare the following \\ four dictionary articles taken from pages 2 and 28 of EKD: \\ acclivity, n., efilukila. \\ acme, n., oxulo. \\ concert, v.t., pangelafana(a) ounongo. \\ conclave, n., eongalo lomeholeko.
}

\title{
Example 1: Presentation of main lemmata
}

\section{Evaluation}

Particularly the mother-tongue speakers of Oshikwanyama will acquire knowledge of how lexical items in English are spelt, and that will assist them in writing correctly. The above representative dictionary articles show that lemma signs are consistently presented in bold print and spelt correctly. EKD serves as an answer to the target users who need guidance or assistance on how English lexical items are spelt.

\section{Sub-lemmata}

\section{Description}

The treatment of sub-lemmata differs from main lemmata in EKD. They are not bolded and they start with capital letters. Compare the following example taken from Page 67 of EKD:

felon, n., omukolokosi. Felony, n., etimba.

gratify, v.t., pandika(e), be pleasing; longe-

la(e) ouna, do a favour to; efela(e)

indulge. Gratitude, n., olupandu. Gratis,

osali, ohali $[\ldots]$

Example 2: Presentation of sub-lemmata

\section{Evaluation}

Example 2 indicates that only the main lemmata, felon and gratify, have been bolded and the sublemmata have not been bolded. In addition, the sub-lemmata Felony, Gratitude and Gratis are started with capital letters each. It has been assumed that this is done due to the fact that there is a full stop that precedes them. All sub-lemmata have been presented as in the example above. This will hinder the user who is looking for the sub-lemmata, because there is no indication to show that that is the sub-lemma. Therefore, the target user might skip it and miss important information presented in the articles of the sub-lemmata.

\section{Parts of speech}

Description 
One of the most fundamental reasons for part of speech indication is that of giving information on the grammatical features and properties of the lemma, giving the user's knowledge of regularities in the grammar of the concerned language (cf. Gouws \& Prinsloo, 2005; Svensén, 2009).

First of all, each lemma sign has been assigned to which part of speech it belongs. That means the part of speech markers follow immediately on the lemma signs. This is not only applied to the main lemmata, but also to the sub-lemmata. Compare the following dictionary articles:

democracy, n., (democratic government) onedi yokupangela osilongo kovańu avese; (country so governed) osilongo sa pange-lwa kovañu avese. Democrat, n. omuniu ou a hala epangelo losilongo li kale momake ovañu avese. felon, n., omukolokosi. Felony, n., etimba.

\title{
Example 3: Parts of speech indicators
}

\section{Evaluation}

If one takes a closer look at the articles in example 3, it can clearly be observed that the main lemmata democracy and felon are indicated as belonging to the category of nouns, that is why the abbreviation $n$ follows them immediately. This is not only applied to the main lemmata, but also to the sub-lemmata. The sub-lemmata Democrat and Felony have also been indicated as belonging to the category of nouns. Therefore, the target users, particularly the Oshikwanyama speaking users who are learning English, will gain knowledge of the parts of speech the lexical items belong to, that will also assist them in using them appropriately in their writing and speaking.

\section{Inconsistency in presenting the parts of speech}

\author{
Description
}

Apart from the fact that all the lemma signs are assigned the parts of speech they belong to, it seems like there is an inconsistency in presenting the parts of speech. Compare the following dictionary articles from Page 54 of EKD:

egg, n., ei, (pl. omai). V.t., hekela(e) mo

on); hongaife(a) (incite); egg on to

(urge

hekela(e) moulai.

elastic, adj., transl. by nanunuka(a), be

ductile. N., ekenja (rubber).

Example 4: Inconsistency in presenting parts of speech indicators

\section{Evaluation}

Based on the above articles, it can be observed that some parts of speech markers have been written in small letters, while some start with capital letters. The parts of speech indicators that have been written with small letters are the ones that follow the main lemma while the ones that are written with capital letters at the beginning are the ones that are preceded by a full stop. They indicate that apart from the part of speech that follows the lemma, the concerned lemma also belongs to another part of speech. Lemma, egg is given its first part of speech as a noun (n), which is written in a small letter, but when it is a verb transitive its parts of speech indicator is in a capital letter (V.t.). This is also applied to the other article, introduced by the lemma elastic. This seems to have been done due to the fact that the part of speech markers that are presented with the capital letters follow a full stop. The parts of speech that are preceded by a full stop in each dictionary article in the dictionary start with a capital letter. The presentation of parts of speech markers has been done inconsistently throughout the dictionary as far as all lemma types are 
concerned. This is not good because it could confuse the target users in their interpretation of the indicators.

Condensing the parts of speech indicators is very important because it saves space in a printed dictionary. However, it should be done consistently.

\section{Indicator of pronunciation}

Description

Pronunciation is not presented in EKD. Usually, it would be indicated between brackets [ ] or slashes //. It should follow immediately after the part of speech indicators (Gouws \& Prinsloo, 2005).

\section{Evaluation}

Pronunciation of the lexical items, especially the lemma signs, is not indicated in the entire dictionary. Therefore, the target users will find it hard, especially the non-speakers of English, which are Oshikwanyama mother-tongue users, in knowing how English lexical items are pronounced. They need to know how a lexical item is pronounced in order for them to acquire experience of pronunciation of foreign language items.

3.2.1.5 Indicator of lemma inflection

\section{Description}

Similar to pronunciation the inflection of lemmata has not been indicated. If a lemma is a noun, it should be indicated how it changes into its plural form. If a lemma is a verb it should be indicated what its present, past or future tense form would be. If it is an adjective, its degrees of comparison should be indicated, especially in a dictionary designed for learners.

\section{Evaluation}

Indicating inflection is very important especially to the target users who are the mother-tongue users of Oshikwanyama because it will assist them in learning English and acquiring vocabulary. This is also important because it will assist them in text production.

\section{The comment on semantics}

This section focuses only on the presentation of translation equivalents.

\section{Translation equivalents}

The evaluation of translation equivalents is done in four categories: the incorrect spelling of translation equivalents, incorrect morphology, obsolete morphology, and incorrect translation equivalents.

\section{The typographical errors of some translation equivalents}

\section{Description}

Some of the translation equivalents that are presented are not spelt correctly. This might be typing errors or because the compilers of EKD are not mother-tongue speakers of Oshikwanyama. Compare the following article from Page 80 of EKD:

gramophone, n., okaxumbo kēngalo.

Example 5: Incorrect spelling of translation equivalent 


\section{Evaluation}

The translation equivalent of the lemma gramophone in example 5 is spelled incorrectly, because in Oshikwanyama there is no such a word as okaxumbo, but there is okaxumba, which is equivalent to the word gramophone in English. The last letter 0 of the okaxumbo should be replaced by letter a for the translation to be correct. Therefore, the correct spelled translation equivalent should be okaxumba, not okaxumbo. Another example from Page 106 is as follows:

liar, n., omufufi, omunoipupulu.

Example 6: Incorrect spelling of translation equivalent

The first translation equivalent in example 6, omufufi, does exist in Oshikwanyama and it is spelt correctly. The second translation equivalent in the above article, omunoipupulu, which is a target language synonym of omufufi, is spelt incorrectly. In Oshikwanyama there is no such word as omunoipupulu, but the correct word should be omunaipupulu. Therefore, the correctly spelled translation equivalent is omunaipupulu. The probable reason behind this is the fact that the compilers of EKD are not mother-tongue speakers of Oshikwanyama; therefore, they might have copied or typed it incorrectly. This is not good for the target users who are not mother-tongue speakers of Oshikwanyama because they will learn an incorrectly spelt word that will hinder them in writing and speaking effectively. Therefore, spelling correctly is needed to avoid giving wrong information to the target users.

Another typing mistake which prevails in EKD is the incorrect spelling of the translation equivalent of the lemma imprison in the following example from Page 93:

imprison, v.t., paka(e) mondolongo, tula(a) mondolongo.

Example 7: Incorrect spelling of translation equivalents

The translation equivalent of the lemma imprison in example 7 is incorrectly spelt because in Oshikwanyama, the word mondolongo does not have letter $n$ between the first letter $o$ and letter $d$ as written in the above article. Therefore, the correct translation equivalents should be paka(e) modolongo, tula(a) modolongo. This will misguide the mother-tongue users of English in pronouncing the translation equivalent correctly. The last example in which the translation equivalents are spelt incorrectly is observed in the following articles, segment and Tuesday from pages 158 and 184 of EKD respectively:

segment, n., ositetele.

Tuesday, n., (efiku) eti vali.

Example 8: Incorrect spelling of translation equivalents

The translation equivalent for the lemma segment is incorrectly spelt due to the reason that there is no such word as ositetele in Oshikwanyama. The correct translation equivalent should be ositetela (oshitetela). In connection with the spelling of the translation equivalent for the lemma Tuesday, it is spelt with the first small letter, which is wrong, because Tuesday is a proper noun. All proper nouns, either in English or in Oshikwanyama, must be capitalised on their first letters .Therefore, the correctly spelt translation equivalent should be Etivali. Overall, however, translation equivalents in EKD are generally spelt correctly.

\section{Incorrect morphology}

Description

Cases of incorrect morphology occur in EKD. Compare the following article from Page 184:

Tuesday, n., (efiku) eti vali. 


\section{Example 9: Incorrect morphology}

\section{Evaluation}

It is not only that the translation equivalent for Tuesday is spelt incorrectly as it is supposed to start with the capital letter as it is a proper noun, but it is also split into two morphemes, which is wrong. This is a single lexical item which refers to one of the week days and it is functioning as a noun. Therefore, it should be written as Etivali, as one lexical item. If the translation equivalent are split incorrectly, it will not only affect the target users in spelling them correctly, but also in pronouncing and using them properly. This might also be a typographic mistake.

\section{Obsolete orthography}

\section{Description}

Apart from the incorrect spelling and incorrect morphological presentation of some translation equivalents, another serious case, which is the obsolete orthography in some of the translation equivalents, is observed. An obsolete orthography is used throughout the dictionary. The following articles from Page 41 of EKD contain obsolete orthography:

delicacy, n., oikulia josikengeli (dainty food). delinquent, n., omunjoni. dell, n., okaniofi (hollow where water

lies in wet season, surrounded by trees).

Example 10: Obsolete orthography in translation equivalents

\section{Evaluation}

In the above three dictionary articles, it can be seen that all the translation equivalents contain the obsolete letters or letter combination, that is, lia, $s i, j$ and $\dot{n}$. This is due to the fact that the orthography that was used around 1954 is no longer in use. It was only contemporary during the time of the production of this dictionary. For example in the first article, the translation equivalent of the lemma delicacy is oikulia josikengeli. In the present orthography the letter combination lia is no longer in use. It has been replaced by the combination lya. This is also the case with the letter $j$ which is replaced by letter $y$, and between letters $s$ and $i$, there should be the letter $h$. Therefore, the correct translation equivalent in the present orthography is oikulya yoshikengeli. In the second article, omunjoni has become omunyoni, and okaniofi has become okanhofi. It is very important to realise that the dictionary is very old and it is less appropriate for modern users, particularly the mother-tongue speakers of English who are the target users of this dictionary, as they will learn orthography that is no longer in use, and their consultation of the dictionary will result in a dysfunctional effect. Therefore, the usefulness of this dictionary is compromised, especially for the English speakers who are learning to write Oshikwanyama. This state of affairs emphasises the urgent need for modern Oshikwanyama dictionaries.

\section{Incorrect translation equivalents}

Description

Apart from obsolete orthography that prevails, there are some cases in which the translation equivalents are partially incorrect. These cases are rare in the dictionary, though. The following article from Page 145 of EKD presents the partially incorrect translation equivalent of the sublemma Rabies.

rabid, adj. transl by v.i., puiduka(a), rage

or by $\mathrm{n}$., omupuidiñu, one madly angry.

Rabies, n., oudu uombua jemuengu.

Example 11: Incorrect translation equivalents 


\section{Evaluation}

The sub-lemma Rabies in example 11 is given a wrong translation equivalent which is oudu uombua jemuengu. This translation equivalent is not correct according to the sub-lemma Rabies, because it is referring to something else. The correct translation equivalent should be eemwengu dombwa. The translation equivalent, oudu uombua jemuengu (yeemwengu) would be translated as the disease of a dog with rabies, which does not make sense and is partially pleonastic. Therefore, if the target users, the speakers of English, come across this translation equivalent, it will misguide or mislead them in learning wrong translation equivalents, which will not help in learning or communicating via Oshikwanyama. The correct translation equivalent of the sublemma should be as follows:

Rabies, n., eemwengu dombwa.

Example 12: Correct translation equivalent

\section{Conclusion}

It has been concluded that no target user for EKD has been clearly defined in the dictionary's front matters. This is vivid in its frame structure, macrostructure as well as its microstructure which, in fact, do not appear to give much emphasis and considerations for any exact user group, but the target user has been defined based on the relevant literature and inference. Another problem in $E K D$ is the obsolete orthography. It is important to realise that the dictionary is very old and it is less appropriate for modern users, particularly the mother-tongue speakers of English who are the target users of this dictionary, as they will learn orthography that is no longer in use, and their consultation of the dictionary will result in a dysfunctional effect. Therefore, the usefulness of this dictionary is compromised, especially for the English speakers who are learning to write in Oshikwanyama. This state of affairs emphasises the urgent need for modern Oshikwanyama dictionaries.

\section{Recommendations}

Based on the critical evaluation made in this study, the business of bilingual dictionary compilation in Namibia, particularly in Oshikwanyama, needs substantial improvement. Very important, at least the effort that was put by the two compilers of EKD cannot, in any way, be unrecognised, since their dictionary serves users with some of their immediate needs. Future compilers of modern dictionaries should adopt the current (orthography) spelling system and use new words that will be used by the modern users of dictionary. The use of typographical and nontypographical structural markers need to be taken into consideration by the future compilers when compiling their dictionaries. This will assist the target users access the correct translation equivalents they are looking for. The paper also recommends that future compilers of dictionaries should make it clear in the outer texts, for instance in the user's guide, the target user groups aimed for the indented dictionary. They should also take into consideration the correct presentation of microstructural aspects, based on the comment on form and the comment on semantics, such as spelling, parts of speech indication, lemma inflection, morphology and translation equivalents.

\section{References}

Bergenholtz, H., \& Gouws, H. R. (2016). On the metalexicographic genre of dictionary reviews, with specific reference to LexicoNordia and Lexikos. Lexikos, 26(1), 60-81.

Bergenholtz, H., \& Tarp, S. (2003). Two opposing theories: On H. E. Wiegand's recent discovery of lexicographic functions. Hermes, Journal of Linguistics, 31(1), 171-196.

Beyer, H. L., \& Augart, J. (2017). From user questions to a basic microstructure: Developing a generative Communication theory for a Namibian German Dictionary. Journal for Studies in Humanities and Social Sciences, 6(2), 1-31. 
Gouws, R. H. (2002). Niching as a Macrostructural Procedure. Lexikos, 12, 133-158.

Gouws, R. H., \& Prinsloo, D. J. (2005). Principles and practice of South African lexicography. Stellenbosch: Sun Press.

Jackson, H. (1996). Dictionary criticism. Unpublished manuscript.

Jackson, H. (2002). Lexicography: An introduction. New York: Routledge.

Klapicová, H. (Ed.). (2005). Composition of the entry in a bilingual dictionary. Retrieved from: https://pdfs.semanticscholar.org/9075/168073ba1941ebc54ed8fd2f54ad359ed99c.pdf

Modjadji, L. A. (2012). Northern Sotho/English bilingual dictionaries: A lexicographic perspective (Unpublished Master's thesis). University of Limpopo, Limpopo, South Africa.

Müller-Spitzer, C. (2013). Textual structures in electronic dictionaries compared with printed dictionaries: A short general survey. In R. H. Gouws, U. Heid, W. Scheweickard \& H. E. Wiegand (Eds.), Dictionaries. An international encyclopaedia of lexicography. supplementary volume: Recent developments with focus on electronic and computational lexicography (pp. 267-381). Berlin: De Gruyter Mouton.

Svensén, B. (2009). A handbook of Lexicography. The theory and practice of dictionary making. Cambridge: Cambridge University Press.

Swanepoel, P. (2013). Evaluation of dictionaries. In R. H. Gouws, U. Heid, W. Scheweickard \& H. E. Wiegand (Eds.), Dictionaries. An international encyclopaedia of lexicography. Supplementary volume: Recent developments with focus on electronic and computational lexicography (pp. 587-596). Berlin: De Gruyter Mouton.

Tarp, S. (2008). Lexicography in the borderland between knowledge and non-knowledge. General lexicographical theory with particular focus on learner's lexicography. Tubingen: Niemeyer.

Tarp, S. (2013). Lexicographic functions. In R. H. Gouws, U. Heid, W. Scheweickard \& H. E. Wiegand (Eds.), Dictionaries: An international encyclopaedia of lexicography. Supplementary volume: Recent developments with focus on electronic and computational lexicography (pp. 460-468). Berlin: De Gruyter Mouton.

Tobias, G. W. R., \& Turvey, B. H. C. (1954). English-Kwanyama Dictionary. Johannesburg: Witwatersrand University Press.

Wang, M. (2012). A critical evaluation of bilingual Chinese/ English dictionaries for elementary and intermediate Mandarin learners at Stellenbosch (Unpublished master's thesis). Stellenbosch University, Stellenbosch, South Africa.

Wiegand, H. E. (1984). On the structure and contents of a general theory of lexicography. In R. R. K. Hartmann (Ed.), Lexeter '83 Proceedings (pp. 13-30). Tübingen: Max Niemeyer Verlag. 